\title{
High-Dose Immunoablative Therapy with Hematopoietic Stem Cell Support in the Treatment of Severe Autoimmune Disease: Current Status and Future Direction
}

\author{
Alan TyNdALL and Takao KoIKE*
}

\begin{abstract}
In the past 5 years approximately 500 patients worldwide suffering from severe autoimmune disease (AD) have received an autologous hematopoietic stem cell transplantation (HSCT) as treatment following high-dose chemotherapy. The EBMT and EULAR data base contains 370 registrations, the most frequently transplanted ADs being multiple sclerosis (MS), systemic sclerosis (SSc), rheumatoid arthritis (RA), juvenile idiopathic arthritis (JIA), systemic lupus erythematosus (SLE) and idiopathic thrombocytopenic purpura (ITP). Around 70\% responded initially well, with durable remission/stabilization seen more frequently in MS and SSc than in RA and SLE, the latter having around $2 / 3$ relapses, the majority of which respond to simple agents. Overall $8 \%$ transplant-related mortality was seen with large inter AD differences $(12.5 \%$ in SSc and only one patient in RA) probably reflecting the degree of vital organ involvement at the time of transplant. This phase $\mathrm{I}$ II data has led to a running phase III randomized trial in SSc called the Autologous Stem cell Transplantation International Scleroderma (ASTIS) trial, and it will soon begin in MS (ASTIMS) and RA (ASTIRA). The concept of immunological "re-setting" has evolved, and needs to be confirmed by longer follow-up and the multicentre, international phase III randomized studies.

(Internal Medicine 41: 608-612, 2002)
\end{abstract}

Key words: transplant, bone marrow, stem cell, autoimmune

\section{Introduction}

The use of high-dose ablative chemotherapy followed by hematopoietic stem cell transplantation (HSCT) in the treatment of severe autoimmune disease (AD) has become the subject of an international project over the past 5 years. Since many of the drugs such as cyclophosphamide (Cy) used to treat severe $\mathrm{AD}$ are limited in their dose by bone marrow toxicity, the concept of exceeding that limit then rescuing the patient with an autologous HSCT grew into an international project along consensus study guidelines such that currently around $500 \mathrm{AD}$ patients have received an HSCT mostly within the context of phase I/II studies (1). This report summarizes these early, in general, positive results, and explains the differences between the various ADs and how this is being translated into phase III prospective randomized controlled studies.

\section{Bone Marrow Transplantation and HSCT}

In the past decade, most transplants have been performed with peripherally harvested hematopoietic stem cells (HSCs), with fewer bone marrow and recently only some cord blood HSC harvesting. From the aspect of treating AD, there are some important differences between peripheral and bone marrow HSC source. Bone marrow contains fewer T cells, but requires a general anesthetic and a longer reconstitution time due to fewer early progenitors. In small children (juvenile idiopathic arthritis), it is sometimes not possible to mobilize enough stem cells for a peripheral harvest: it is known that at least $2 \times 10^{6}$ CD34 positive cells (stem and early progenitors) per kg body weight are needed for reliable hematological reconstitution.

On the other hand, a peripheral HSC source requires mobilization of stem cells from the marrow using high-dose Cy and/ or growth factors, mostly G-CSF. For AD patients, this raises some new aspects. In 5 patients, the first attempts to mobilize stem cells were unsuccessful, probably due to previous marrow toxic treatments. This was not as common as initially anticipated (2) and was overcome by combining the graft product with a second mobilization, or moving to a bone marrow harvest. Also, G-CSF is a potential trigger for a flare of $\mathrm{AD}$, and this was observed in some patients, especially in RA, in 8 out of 72 patients (3). Most of these flares were relatively easy to control, but in at least one (multiple sclerosis) a fatal outcome ensued (4). In some cases the Cy in doses from $2-4 \mathrm{~g} / \mathrm{m}^{2}$ given as mobilization resulted in a rapid improvement of the $\mathrm{AD}$, such that this was adopted as the pre-randomization step in phase III studies for RA and SLE (see below).

Concerning purging, an association between severity of purging and infection risk was seen, without clear advantage (5). It

From Department of Rheumatology, University of Basel, Switzerland and *Department of Medicine II, Hokkaido University School of Medicine, Sapporo Reprint requests should be addressed to Dr. Alan Tyndall, Felix Platter Spital, Burgfelderstrasse 101, 4012; Basel, Switzerland 
is known that complete cellular purge either in vivo or in vitro is not possible with chemotherapy or radiation, so that at the most an "autoimmune debulking"occurs with autologous HSCT. Overall a concept of resetting the immune auto reaction rather than ablating and inducing tolerance, as was suggested by animal model experiments, has evolved with this project.

\section{Animal Models}

The first publications in this area were from Denman et al in 1969 showing passive transfer of SLE from NZW mouse model to resistant strains (6), and later confirmed by Morton and Siegel (7). The subject has since been extensively reviewed $(8,9)$ and it formed part of the original impetus to move to human phase I/II studies.

\section{Coincidental Autoimmune Disease and HSCT}

Since the first reports of improvement and even long-term remission of RA following allo HSCT, many retrospective and some prospective case reports and series have been published (10). A degree of reporting bias makes interpretation difficult.

\section{Results in Human Autoimmune Disease HSCT}

The European Group for Blood and Marrow Transplants (EBMT) and the European League Against Rheumatism (EULAR) data base contains data on 370 registrations, the most commonly transplanted diseases being MS, systemic sclerosis (SSc), RA, JIA and SLE (Table 1). The data comes from 82 transplant centers in 21 countries and the overall actuarially adjusted transplant related mortality (TRM) was $8 \%$ (11). There was a marked difference between AD groups with a TRM of $12.5 \%$ in SSc and only one patient with RA, probably reflect-

Table 1. Numbers of Autoimmune Diseases Receiving HSCT (EBMT/EULAR Data Base)

\begin{tabular}{lrlr}
\hline Multiple sclerosis & 109 & MCTD & 4 \\
Myasthenia & 1 & Cryoglobulinemia & 3 \\
Neuropathy & 1 & Behçet & 3 \\
ALS & 2 & Wegener's & 3 \\
SSc & 63 & Polychondritis & 1 \\
SLE & 33 & ITP & 10 \\
Rheumatoid arthritis & 70 & AIHA & 3 \\
Psoriatic arthritis & 2 & PRCA & 4 \\
Juvenile idiopathic arthritis & 43 & Evans & 2 \\
Ankylosing spondilitis & 2 & TTP & 2 \\
Sjögren & 1 & Bowel disease & 2 \\
Vasculitis & 1 & Other & 3 \\
Dermatomyositis & 7 & & \\
\end{tabular}

ALS: amyotrophic lateral sclerosis, MCTD: mixed connective tissue disease, ITP: idiopathic thrombocytopenic purpura, AIHA: autoimmune haemolytic anaemia, PRCA: pure red cell aplasia, TTP: thrombotic thrombocytopenic purpura. ing degree of vital organ involvement. Also different were response rates and types. In RA, JIA and SLE more patients responded early but later relapsed than for MS and SSc.

\section{Multiple Sclerosis}

An analysis of the first 85 MS patients showed a 3-year progression-free survival of $78 \%$ of the secondary progressive cases, with a risk of death from any cause at three years being $10 \pm 7$ months, $95 \%$ confidence interval (12). The median follow-up was 16 (range: 3-59) months. Fewer positive responses were seen in primary progressive MS $(66 \% \pm 23 \%)$.

A more impressive effect was seen with the MRI changes, considered a surrogate marker of MS. In a ten patient subgroup analysis from one center, the gadolinium enhancing MRI lesions were reduced to zero after a median 15 (range: 4-30) month follow-up (13). However, more long-term follow-up is necessary in this chronic disease, including a randomised controlled trial (see ASTIMS Trial below).

The majority of MS cases received a peripheral stem cell transplant, being mobilized with Cy (mostly $4 \mathrm{~g} / \mathrm{m}^{2}$ ) and GCSF. There was a suggestion that G-CSF alone could induce a flare of the MS, being less when combined with Cy. Conditioning was mostly with BEAM (carmustine, etoposide, cytosine-arabinoside and melphalan) alone (16\%) or BEAM plus ATG (47\%). Other regimens included Cy plus TBI and busulphan/Cy. No clear advantage of $\mathrm{T}$ cell purging was observed.

\section{Systemic Sclerosis (SSc)}

In the first 45 patients, an improvement of $25 \%$ or more was seen in $70 \%$ of the patients, with a TRM of $17 \%$ (14). Several protocols were used, mostly either Cy based $4 \mathrm{~g} / \mathrm{m}^{2}$ Cy mobilization and Cy $200 \mathrm{mg} / \mathrm{kg}$ body weight conditioning or radiation $8 \mathrm{~Gy} / \mathrm{Cy}$ plus Cy $120 \mathrm{mg} / \mathrm{kg}$ body weight. With further patient recruitment, the TRM fell to $12.5 \%$, which was considered to be related to more careful patient selection. Lung function tended to stabilize and some factors were identified as potentially hazardous for HSCT, eg pulmonary hypertension $>50 \mathrm{mmHg}$ mean pulmonary arterial pressure, severe cardiac involvement, severe pulmonary fibrosis and uncontrolled systemic hypertension. When such patients were excluded from the analysis, the TRM was 7\%, suggesting that the proposed randomized controlled trial (see ASTIS Trial below) would be ethical, given the near 50\% 5-year mortality of this subgroup of patients.

\section{Rheumatoid Arthritis}

An analysis of the first 51 of 63 transplanted patients showed significant improvement, with $78 \%$ achieving an ACR 50 response. This is a composite score of clinical and laboratory parameters which should improve by at least $50 \%$. The median follow-up was 18 (range: 6-40) months, and the majority of patients received a conditioning regimen of Cy $200 \mathrm{mg} / \mathrm{m}^{2}$ 
alone and received peripherally harvested stem cells after either G-CSF or Cy/G-CSF (equal numbers) mobilization. Most of the patients had failed at least 3 conventional disease modifying antirheumatic drugs (DMARDs) such as methotrexate, leflunamide or sulphasalazine before the transplant. In addition some patients had also failed anti-TNF alpha treatment. Some degree of relapse was seen in $73 \%$ of patients post transplant, but in the majority it was relatively easy to control with drugs which had proven ineffective pretransplant (EBMT/ EULAR registry report in progress). These phase I/II experiences have been integrated into a phase III randomized studyASTIRA Trial, see below.

\section{Juvenile Idiopathic Arthritis}

Forty-three children with juvenile idiopathic arthritis (JIA), mostly the systemic form called Stills disease, were registered. Most of these cases were treated in two Dutch centers using a bone marrow obtained stem cell source and a conditioning protocol of Cy $200 \mathrm{mg} / \mathrm{kg}$ body weight, TBI $4 \mathrm{~Gy}$ and ATG (15). Overall there were 15 complete remissions and 3 partial remissions reported. In those attaining remission, the corticosteroid dose could be reduced and some patients experienced puberty and catch-up growth. Three patients died from macrophage activation syndrome, thought to be related to intercurrent infection or uncontrolled systemic activity of the disease at the time of transplantation. Protocols were modified accordingly such that systemic activity is controlled before the transplant with methyl prednisolone intravenously. Since this modification, no further such deaths have occurred. Further phase I/ II pilot studies will be undertaken before the optimal phase III randomised study will be proposed (16).

\section{Systemic Lupus Erythematosus}

Of the 29 registrations, most had either renal and/or CNS involvement, and 21 had failed conventional Cy treatment. A peripheral stem cell source after mobilization with $\mathrm{Cy}$ and GCSF was used in the majority. Fifteen patients received a conditioning with $\mathrm{Cy}$ and ATG, and five other regimens were employed. There were 4 deaths due to treatment and one from progressive disease, resulting in an actuarially adjusted TRM of 14 (range: $1-27) \%$.

In those patients with sufficient data for analysis, 16 achieved a "remission", defined as a SLEDAI (Systemic Lupus Erythematosus Disease Activity Index) of $\leqq 3$ and steroid reduction to $<10 \mathrm{mg} /$ day. Six others did not reach this end point. Half of the remissions relapsed to some degree, and were mostly easily controlled on standard agents which had previously been ineffective. Better results are seen with single center/single protocols (17). A more complete analysis of the data is underway with a view to proposing the most appropriate phase III protocol (ASTIL).

\section{Refractory Autoimmune Cytopenias}

A preliminary review of 16 cases has been reported (Jakob Passweg on behalf of the EBMT autoimmune disease working party) consisting of the following diseases: idiopathic thrombocytopenic purpura $(n=9)$, pure red cell aplasia $(n=4)$, autoimmune hemolytic anemia $(n=2)$ and Evans Syndrome $(n=1)$. Median age was 31 (range: 4-45) years and median time to transplantation was 93 months (12-236). Most received stem cells from blood mobilized with either growth factors $(n=7)$ or Cy plus growth factors $(n=6)$. There were two bone marrow harvests. Conditioning regimens included Cy alone $(n=3), C y$ with other drugs or ATG $(n=9)$, melphalan $(n=2)$ or were fludarabine based $(n=2)$. Most $(\mathrm{N}=12)$ had purging of immune cells from the graft product. Median follow-up of surviving patients was 30 months (5-53). Three patients died within 100 days post transplantation, two of hemorrhage and one from progressive hemolysis. Eight patients showed a response to treatment, 4 complete remissions (2 ITP, 1 Evans and 1 PRCA), sustained in three patients. Randomised trials are under discussion for ITP.

The numbers of cases with vasculitis, Behçet's disease, relapsing polychondritis and other ADs are too small to draw meaningful conclusions, with further phase I/II standardized protocol pilot studies proceeding.

\section{Japanese Experience}

Several centers in Japan have been pursuing HSCT in AD. For adult patients with $\mathrm{AD}$, the author's group has recently performed HSCT mainly for diffuse type SSc, with striking clinical improvement. Among SSc patients who visited Hokkaido University Hospital in Sapporo, three patients were finally indicated for HSCT, as assessed by ASTIS-based inclusion and exclusion criteria. The patients' age ranged from 19 to 57 at the time of HSCT, and the disease duration was one to three years. Autologous peripheral HSCs were mobilized by G-CSF alone or Cy plus G-CSF, and CD34-positive cell selection was performed on the graft product in all cases. Cy 200 $\mathrm{mg} / \mathrm{kg}$ in 4 doses was used for conditioning followed by HSCT.

When assessed at day 90 after transplantation, significant improvement of skin sclerosis was observed, as assessed by modified Rodnan's total skin score (32-76\% reduction). Improvement of skin sclerosis was first observed even after initial Cy administration for peripheral HSC mobilization, suggesting a direct effect of the reagent on the skin lesions in SSc, in addition to the effect of immune system reconstitution. Prolonged improvement of skin sclerosis has been observed for two years or more in the first two cases, which should be further continuously evaluated. During the course of treatment, in addition to some infectious episodes, hemophagocytic syndrome and pulmonary capillary leak syndrome were observed. Although these were successfully treated by corticosteroid therapy, it was suggested that patients with SSc might present specific problems that must be carefully monitored, even though 
the organ involvement was apparently limited.

HSCT has also been applied for some cases of JIA with severe clinical courses. One of the earliest reports was a 15year-old JIA patient with refractory history of seven years (18). After conditioning by $200 \mathrm{mg} / \mathrm{kg}$ of Cy in 4 doses with ALG and prednisolone, followed by HSCT with purified autologous CD34-positive cell, the patient has been free from immunosuppressive therapy for more than one year. Another report in Japan was from Osaka Medical Centre, which included initially two cases of HSCT with severe and refractory JIA. Four children with JIA have received HSCT, including two autologous peripheral stem cells, one bone marrow cells and one cord blood cells as sources of HSCs. Complete or partial disease remission was obtained after conditioning by using VP-16 and thiotepa combined with ALG, followed by peripheral CD34 cells grafting.

Experiences of HSCT for AD is still limited in Japan. This is due to the rather short history of this approach in the field of rheumatology, despite the fact that HSCT is broadly used for the treatment of hematological disorders. Since the advantages of HSCT for AD have recently been established, this new therapeutic approach to intractable $\mathrm{AD}$ should be considered.

\section{Randomized, Prospective Controlled Trials}

\section{ASTIS (Autologous Stem cell Transplantation International Scleroderma) Trial}

Diffuse skin SSc (scleroderma) patients are selected who have less than 4 years of diffuse skin involvement and evidence of progressive and organ or life threatening disease. The primary end point on which the trial is powered is event-free survival at 2 years, events being arbitrarily but precisely defined to capture irreversible and severe end organ failure or death. Exclusion criteria are based on the phase I/II data to avoid an unacceptably high TRM risk together with a minimal chance of clinically significant improvement. The treatment arm is mobilization with Cy $4 \mathrm{~g} / \mathrm{m}^{2}$ and G-CSF, followed by CY 200 $\mathrm{mg} / \mathrm{kg}$ body weight conditioning plus ATG and a CD 34 selected graft. The control arm is monthly IVI pulse CY $750 \mathrm{mg} /$ $\mathrm{m}^{2}$ for 12 months. Each arm will have 100 patients.

ASTIS is ongoing, and further details are available (see website: www.astistrial.com).

\section{ASTIMS (Autologous Stem cell Transplantion International Multiple Sclerosis) Trial}

Secondary progressive MS patients with an EDSS score of between 3.5 and 6.5 will be randomized to either HSCT (BEAM and ATG followed by an unmanipulated graft reconstitution) or the control arm, probably mitoxantrone. The primary end point is progression free survival at 3 years, and each arm will recruit 80 patients. Further details are available on the EBMT website (www.ebmt.org).

\section{ASTIRA (Autologous Stem cell Transplantation International Rheumatoid Arthritis) Trial}

Active RA patients who have failed at least 4 DMARDS including methotrexate and anti TNF alpha programs with a disease duration of between 2-15 years will all receive stem cell mobilization with Cy $4 \mathrm{~g} / \mathrm{m}^{2}$ and G-CSF. Randomization will then occur to either continue conventional therapy with either methotrexate or leflunamide or conditioning with Cy 200 $\mathrm{mg} / \mathrm{m}^{2}$ and ATG. The graft will not be manipulated, and maintenance with methotrexate or leflunamide will be given. The primary end point is the number of patients reaching a good or moderate EULAR response and/or ACR 20 at six months. Sixteen patients in each arm are required, calculated on a $>50 \%$ difference in the two groups. For further details, see the EBMT website (www.ebmt.org).

\section{Conclusion}

The phase I/II data suggest that for some highly selected AD patients HSCT could offer another treatment option with an ethically acceptable benefit/risk ratio. However it will only be through prospective, multicenter, randomized controlled trials that this suggestion could become evidence based. Such trials are more complicated and prone to recruitment problems, since no one product or company is involved, but rather a principle. This could and should be a major goal.

\section{References}

1) Tyndall A, Gratwohl A. Blood and marrow stem cell transplants in autoimmune disease. A consensus report written on behalf of the European League Against Rheumatism (EULAR) and the European Group for Blood and Marrow Transplantation (EBMT). Br J Rheumatol 36: 390-392, 1997.

2) Marmont A, Tyndall A, Gratwohl A, Vischer T. Haematopoietic precursor-cell transplants for autoimmune diseases. Lancet 345: 975, 1995.

3) Snowden JA, Biggs JC, Milliken ST, Fuller A, Brooks PM. A phase I/II dose escalation study of intensified cyclophosphamide and autologous blood stem cell rescue in severe, active rheumatoid arthritis. Arthritis Rheum 42: 2286-2292, 1999.

4) Openshaw $\mathrm{H}$, Stuve $\mathrm{O}$, Antel JP, et al. Multiple sclerosis flares associated with recombinant granulocyte colony-stimulating factor. Neurology 54: 2147-2150, 2000.

5) Kashyap A PJ, Tyndall A. Autologous hematopoietic stem cell transplant regimens for the treatment of severe autoimmune diseases. in: Autologous Blood and Marrow Transplantation: Proceedings of the Tenth International Symposium Dallas, Texas. A DKaK, Ed. Charlottesville, Virginia: Carden Jennings Publishing; 2001: 219-225.

6) Denman AM, Russell AS, Denman EJ. Adoptive transfer of the diseases of New Zealand black mice to normal mouse strains. Clin Exp Immunol 5: 567-595, 1969.

7) Morton JI, Siegel BV. Transplantation of autoimmune potential. I. Development of antinuclear antibodies in $\mathrm{H}-2$ histocompatible recipients of bone marrow from New Zealand Black mice. Proc Natl Acad Sci USA 71: 2162-2165, 1974.

8) Marmont AM. New horizons in the treatment of autoimmune diseases: immunoablation and stem cell transplantation. Annu Rev Med 51: 115134, 2000.

9) Knaan-Shanzer S, Houben P, Kinwel-Bohre EP, van Bekkum DW. Remission induction of adjuvant arthritis in rats by total body irradiation and autologous bone marrow transplantation. Bone Marrow Transplant 8: 333-338, 1991.

10) Tyndall A, Gratwohl A. Immune ablation and stem-cell therapy in autoimmune disease. Clinical experience. Arthritis Res 2: 276-280, 2000.

11) Tyndall A, Passweg J, Gratwohl A. Haemopoietic stem cell transplantation in the treatment of severe autoimmune diseases 2000. Ann Rheum 
TYNDALL and KoIKE

Dis 60: 702-707, 2001.

12) Fassas A, Passweg J, Anagnostopoulos A, et al. Hematopoietic stem cell transplantation for multiple sclerosis: a retrospective multicentre study. J Neurology 2002 (in press).

13) Mancardi GL, Saccardi R, Filippi M, et al. Autologous hematopoietic stem cell transplantation suppresses Gd-enhanced MRI activity in MS. Neurology 57: 62-68, 2001.

14) Binks M, Passweg JR, Furst D, et al. Phase I/II trial of autologous stem cell transplantation in systemic sclerosis: procedure related mortality and impact on skin disease. Ann Rheum Dis 60: 577-584, 2001.

15) Wulffraat NM, Sanders LA, Kuis W. Autologous hemopoietic stem-cell transplantation for children with refractory autoimmune disease. Curr
Rheumatol Rep 2: 316-323, 2000.

16) Barron KS, Wallace C, Woolfrey CEA, et al. Autologous stem cell transplantation for pediatric rheumatic diseases. J Rheumatol 28: 2337-2358, 2001.

17) Traynor AE, Schroeder J, Rosa RM, et al. Treatment of severe systemic lupus erythematosus with high-dose chemotherapy and haemopoietic stem-cell transplantation: a phase I study. Lancet 356: 701-707, 2000.

18) Nakagawa R, Kawano $Y$, Yoshimura E, et al. Intense immunosuppression followed by purified blood CD34+ cell autografting in a patient with refractory juvenile rheumatoid arthritis. Bone Marrow Transplant 27: 333$336,2001$. 